\title{
Lack of efficacy of pomegranate supplementation for glucose management, insulin levels and sensitivity: evidence from a systematic review and meta-analysis
}

Haohai Huang ${ }^{1 *+}$ (D), Dan Liao ${ }^{2+}$, Guangzhao Chen ${ }^{3}$, Honglang Chen ${ }^{4}$ and Yongkun Zhu ${ }^{1}$

\begin{abstract}
Background: The potential glucose-lowering effects of pomegranate have been reported in animal and observational studies, but intervention studies in humans have generated mixed results. In this paper, we aimed to conduct a systematic review and meta-analysis of randomized controlled trials (RCTs) to evaluate the precise effects of pomegranate supplementation on measures of glucose control, insulin levels and insulin sensitivity in humans.

Methods: Comprehensive electronic searches were conducted in PubMed, Embase, and the Cochrane Library. Studies included were RCTs that evaluated the changes in diabetes biomarkers among adults ( $\geq 18$ years) following pomegranate interventions. The predefined outcomes included fasting blood glucose (FBG), fasting blood insulin (FBI), glycated haemoglobin ( $\mathrm{HbA1c}$ ), and homeostatic model assessment of insulin resistance (HOMA-IR). Endpoints were calculated as weighted mean differences (WMDs) with 95\% confidence intervals (Cls) by using a random-effects model. Publication bias, subgroup analyses, sensitivity analysis and random-effects meta-regression were also performed to explore the influence of covariates on the net changes in fasting glucose and insulin concentrations.
\end{abstract}

Results: Sixteen eligible trials with 538 subjects were included. The pooled estimates suggested that pomegranate did not significantly affect the measures of FBG (WMD, $-0.6 \mathrm{mg} / \mathrm{dL} ; 95 \% \mathrm{Cl},-2.79$ to $1.58 ; P=0.59)$, FBI $(\mathrm{WMD}, 0.29 \mu \mathrm{Ul} / \mathrm{mL} ; 95 \% \mathrm{Cl}$, -1.16 to $1.75 ; P=0.70$ ), HOMA-IR (WMD, $-0.04 ; 95 \% \mathrm{Cl},-0.53$ to $0.46 ; P=0.88)$ or HbA1c (WMD, $-0.11 \% ; 95 \% \mathrm{Cl},-0.39$ to -0 . 18; $P=0.46)$. Overall, significant heterogeneity was detected for $\mathrm{FBI}$ and HOMA-IR, but subgroup analysis could not identify factors significantly influencing these parameters. These results were robust in sensitivity analysis, and no significant publication bias was found in the current meta-analysis.

Conclusion: Pomegranate intake did not show a notably favourable effect on improvements in glucose and insulin metabolism. The current evidence suggests that daily pomegranate supplementation is not recommended as a potential therapeutic strategy in glycemic management. Further large-scale RCTs with longer duration are required to confirm these results.

Keywords: Pomegranate, Glucose, Insulin, Diabetes mellitus, Meta-analysis

\footnotetext{
* Correspondence: haohaihuang@hotmail.com

${ }^{\dagger}$ Equal contributors

'Department of Clinical Pharmacy, Dongguan Third People's Hospital,

Affiliated Dongguan Shilong People's Hospital of Southern Medical

University, Dongguan, Guangdong, China

Full list of author information is available at the end of the article
}

\section{Biomed Central}

(c) The Author(s). 2017 Open Access This article is distributed under the terms of the Creative Commons Attribution 4.0 International License (http://creativecommons.org/licenses/by/4.0/), which permits unrestricted use, distribution, and reproduction in any medium, provided you give appropriate credit to the original author(s) and the source, provide a link to the Creative Commons license, and indicate if changes were made. The Creative Commons Public Domain Dedication waiver (http://creativecommons.org/publicdomain/zero/1.0/) applies to the data made available in this article, unless otherwise stated. 


\section{Background}

Diabetes mellitus is one of the most important public health challenges with an enormous economic burden worldwide. The estimated number of diabetic patients worldwide was 366 million in 2014, and it is projected to rise to 552 million by 2030 [1]. Glycemic control presents a constant challenge for patients with diabetes. Poor glycemic management causes long-term adverse outcomes in individuals with diabetes mellitus, including micro- and macrovascular complications such as myocardial infarction and stroke, renal failure, blindness and peripheral neuropathy [2-4]. Subjects with impaired fasting glucose (IFG) or impaired glucose tolerance (IGT) are usually considered to have a high future risk of developing diabetes [5]. Moreover, prospective cohort studies in subjects without diabetes have also revealed that increased insulin resistance worsened glycemic control and contributed to the development of type 2 diabetes mellitus (T2DM) [6-8]. Appropriate management of hyperglycaemia is thought to decrease the complications and morbidity of diabetes. Given its high disability and mortality rates, identifying modifiable lifestyle factors is important in primary, secondary, and tertiary prevention in patients with diabetes and healthy people. In recent decades, lifestyle interventions, including dietary micronutrients or functional food supplementation have generally been used to improve glycemic levels and have been incorporated into guidelines for the prevention and treatment of diabetes [9-11].

Pomegranate (Punica granatum L.) has a high concentration of antioxidants and bioactive polyphenols, and it has therefore been widely investigated for its antioxidant, antiinflammatory, anti-atherogenic, and anti-hyperglycemic effects [12, 13]. Fresh pomegranate juice (PJ) is rich in phenolic acids (including gallic acid, caffeic acid, chlorogenic acid, ferulic acid, and coumaric acids), non-phenolic acids, citric acid, succinic acid, malic acid, oxalic acid, and ascorbic acid [14, 15]. Pomegranate seed oil (PSO) consists of approximately $80 \%$ conjugated linolenic acid, (9-cis, 11trans, 13-cis) octadecatrienoic acid or punicic acid [16]. Pomegranate extract (PE) contains abundant anthocyanins, punicalin, pedunculagin, punicalagin, gallagic acid and ellagic acid [17]. Accumulating evidence indicates that pomegranate fractions from different parts of the fruit have been used to prevent and treat a wide range of diseases, including CVD, hypertension, obesity, and diabetes [18-20]. Encouraging findings from experimental research have indicated that pomegranate juice or pomegranate extracts improve glycemic metabolism, lower insulin requirements, and ameliorate insulin sensitivity [21-23]. Several observational studies have also suggested that pomegranate consumption was associated with improved glycemic control or a decreased risk for diabetes [24-26]. However, the precise effects of pomegranate on insulin and glucose metabolism in humans are inconsistent, and its optimal role in the clinical management of hyperglycaemia has not been fully established [27-32].

We performed a systematic review and meta-analysis of randomized controlled trials (RCTs) to assess the potential role of pomegranate treatment in the management of glycemic control, insulin levels and insulin sensitivity in comparison with placebo or other interventions among adults ( $\geq 18$ years). Our primary outcomes were the differences in the levels of fasting blood glucose (FBG) and fasting blood insulin (FBI). Secondary outcomes included glycated haemoglobin (HbA1c) and homeostatic model assessment of insulin resistance (HOMA-IR).

\section{Methods}

\section{Study eligibility criteria}

Studies were eligible for inclusion in this review if they met the following criteria: 1) Study participants: adult male and female participants (age $\geq 18 \mathrm{y}$ ) with or without co-morbidities (such as hypertension, diabetes, or peripheral arterial disease) were included. 2) Types of interventions: participants needed to have specifically ingested a pomegranate intervention (no matter which type or regimen applied) for $\geq 1$ week. Studies in which pomegranate was combined with other interventions (e.g., taking glucose-lowering drugs) were included when the control group received the same treatment. 3) Comparators: placebo or other interventions were used. 4) Outcome measures: studies reported data on at least one of the following endpoints: FBG, FBI, HbA1c, or HOMA-IR. In addition, the initial or endpoint values for the outcomes or their differences and their SD or SE or the $95 \%$ CI of each group were available. 5) Study design: each study was an RCT in humans with either a parallel or crossover design.

\section{Data sources and search strategy}

We conducted and reported the present systematic review and meta-analysis following the PRISMA statement for the identification, screening, eligibility, and inclusion of articles [33]. Medline (http://www.ncbi.nlm.nih.gov/ pubmed), Embase (http://www.embase.com), and the Cochrane Library (http://www.cochrane.org) databases were systematically searched for eligible studies from inception to February 28, 2017. Additionally, we also searched Google Scholar and ClinicalTrials.gov (https:// clinicaltrials.gov/) to identify other potentially eligible trials. The structured search strategies used the following search key words and Medical Subject Headings (MeSH) terms: (pomegranate OR Punica) AND (glycemic control OR glycaemic control OR glucose control OR glycaemic OR glucose OR blood sugar OR blood glucose OR fasting plasma glucose OR FBG OR glucose tolerance OR 
insulin resistance OR insulin OR blood insulin OR fasting blood insulin OR insulin sensitivity OR FBI OR Haemoglobin A1c OR HbA1C OR glycated haemoglobin OR glycosylated haemoglobin OR homeostatic model assessment of insulin resistance OR HOMA-IR OR diabetes mellitus OR diabetes) AND sensitivity RCT filters (the specific and sensitive strategies developed to ensure optimal collection of RCTs in electronic searches). The search was restricted to English-language publications and clinical trials conducted in human subjects. Citation tracking was also performed on relevant review articles and editorials, and the reference lists of all the included studies were cross-referenced to ensure completeness. Two investigators (H.C. and D.L.) independently performed the literature searches. Any disagreements were resolved through discussion to reach consensus. After the removal of duplicate studies, we screened the titles and abstracts for relevance and accessed the full texts to identify the eligibility of the studies for inclusion in this meta-analysis.

\section{Data extraction}

Data extraction was performed by D.L. and was confirmed independently by two other authors (H.H. and H. C.). Extracted data were entered into a predefined standardized Excel (Microsoft Corporation, USA) file. We also sought the supplementary files of the included trials or contacted the corresponding authors to verify the extracted data and to request any missing data. From each eligible trial, the following information was extracted: 1) Study characteristics, including first authors, publication year, sample size, study design, study duration, dose, type of intervention, and outcome measures. 2) Participants' information, including mean age, sex, body mass index (BMI), baseline health status and baseline FBG. When the same patients were reported in several publications, we retained only the largest study to avoid duplication of information. For trials with more than one intervention group (e.g., with different doses of pomegranate), multiple comparisons were considered. The primary outcome measures were the net changes in FBG and FBI concentrations, and the secondary outcomes included changes in HbA1c and HOMA-IR. All values were captured as the means \pm SD and converted to $\mathrm{mg} / \mathrm{dL}$ for glucose and $\mu \mathrm{IU} / \mathrm{mL}$ for insulin using the following conversion factors: $1 \mathrm{mmol} / \mathrm{L}=18 \mathrm{mg} / \mathrm{dL}$ for glucose and $1 \mathrm{pmol} / \mathrm{L}=6.945 \mu \mathrm{IU} / \mathrm{mL}$ for insulin value.

\section{Assessment of methodological quality}

We used the Cochrane risk of bias tool to evaluate the risk of bias in the methodological quality of the included trials [34]. The assessment of quality characteristics used the following criteria: 1) sequence generation of allocation; 2) allocation concealment; 3) masking of participants and personnel; 4) blinding of outcome assessors; 5) incomplete outcome data; 6) selective outcome reporting; and 7) other sources of bias. Each item was judged to be at low, unclear, or high risk of bias, based on whether the level of bias in the domains may have led to material bias in the outcomes of interest. Trials with high risk of bias for any one or more key domains were considered to be at high risk of bias, while trials with low risk of bias for all key domains were considered to be at low risk of bias; otherwise, studies were considered to be at unclear risk of bias.

\section{Grading quality of evidence}

Two reviewers (H. H. and D. L.) independently evaluated the quality of the evidence for the primary and secondary outcomes according to the GRADE methodology for risk of bias, inconsistency, indirectness, imprecision, and publication bias; they rated each as very low, low, moderate, or high. If disagreements occurred between the two reviewers, a third author would make the decision through discussion. Summary tables were constructed using the GRADE Profiler (GRADEpro, version 3.6).

\section{Statistical analysis}

In the present meta-analysis, weighted mean difference (WMD) and 95\% confidence interval (CI) were used as the main measures to summarize the clinical effect of the arms on the outcomes. We performed $I^{2}$ testing to assess the magnitude of the heterogeneity between trials; values greater than $50 \%$ were regarded as being indicative of moderate-to-high heterogeneity [35]. We pooled outcome data using a random-effects model accounting for clinical heterogeneity [36]. To explore the possible influence of covariates on net changes, pre-specified subgroup analyses were conducted to evaluate the effects of the following factors on primary outcomes: pomegranate-product types, study design, baseline FBG, study duration, health status and initial BMI measurements of the participants. In addition, we further explored our findings using two additional sensitivity analyses. To assess the potential impact of the quality of the studies on the outcomes, we performed a sensitivity analysis with the exclusion of low-quality studies. To test the robustness of the findings, we also conducted a sensitivity analysis, which was investigated using the leave-one-out approach (omitting one study at each turn and repeating the analysis). We plotted the SEs of the studies against their corresponding effect sizes to examine potential publication biases in the meta-analysis. Publication bias was assessed by visually inspecting a funnel plot and by using the Egger test [37]. A $P$ value of $<0.05$ was considered statistically significant for all analyses. All statistical analyses were performed using STATA (version 12; StataCorp, College Station, TX). 


\section{Meta-regression analysis}

As potential confounders of treatment effects, dosage and duration of supplementation with pomegranate juice were applied by a restricted maximum likelihood (REML) based meta-regression analysis to explore their association with the estimated effect size in glucose outcome [38]. The analysis was also performed by using STATA version 12.0 (Stata Corporation LP, College Station, TX, USA). This method corresponds to random-effects meta-regression including both within-study variances of treatment effects and the residual between-study heterogeneity.

\section{Results}

\section{Identification of relevant studies}

Fig. 1 shows the details of our literature screening, study selection, and reasons for exclusion. The initial search yielded 139 potentially relevant citations. After the removal of duplicates, 102 titles and abstracts were screened; of these, 78 were excluded because they were clearly not relevant to our meta-analysis. The full-text publications were obtained for the remaining 24 articles. A total of 10 articles were subsequently excluded for the reasons listed in Fig. 1. Subjects in one study were also divided into 2 subgroups on the basis of different doses of pomegranate ellagitannin extract consumption used
(710 mg/day intake subgroup and $1420 \mathrm{mg} /$ day intake subgroup) [39]. The work conducted by Fuster-Munoz et al. was also separated into 2 subgroups: the pomegranate juice and the pomegranate juice diluted 1:1 with water subgroups [40]. Finally, a total of 16 RCTs that met our inclusion criteria were included in the present pooled analysis [27-32, 39-46].

\section{Study characteristics}

A summary of the study characteristics included in the meta-analysis are presented in Table 1 . Sixteen trials with a total of 627 subjects were included in the metaanalysis. The total number of subjects included in each study ranged from 14 to 74 subjects. Among the included studies, fifteen studies reported the FBG outcome $[27,28,30-32,39-46], 8$ reported FBI values [29-31, 41-43, 45, 46], 3 reported HbA1c [27, 41, 43] and 7 reported HOMA-IR [29, 31, 41-43, 45, 46]. The mean age of participants in each trial ranged from 30 to 70 years, with differing age ranges in most studies. Of the 16 trials included in the current meta-analysis, 11 studies used pomegranate juice as a supplement (the dosage ranged from 120 to $500 \mathrm{ml} /$ day) [27, 28, 30-32, 40, 41, 44-46], 2 studies used pomegranate seed oil as treatments (the dosage ranged from 400 to $2000 \mathrm{mg} /$ day) $[29,43]$, and 3

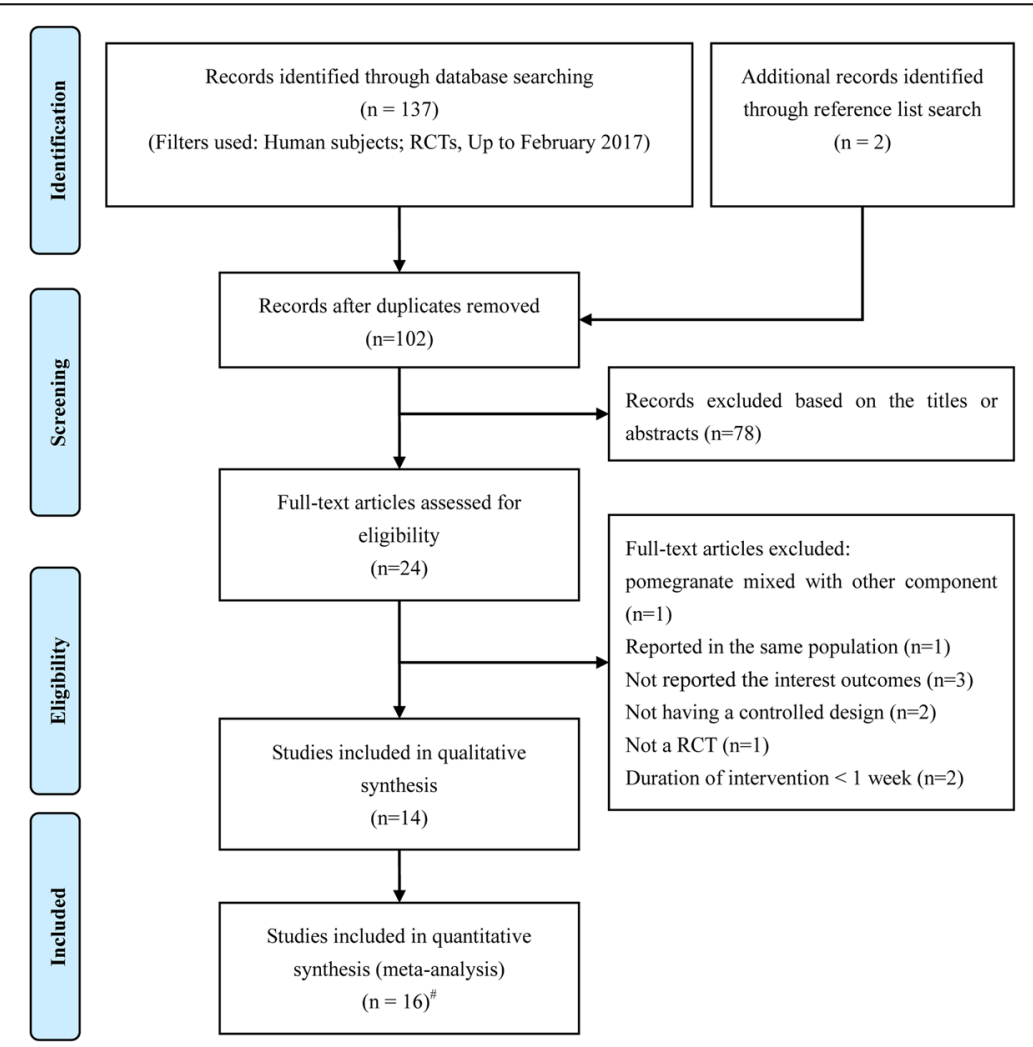

Fig. 1 Flow chart of data base searches and articles included in the present meta-analysis. " The work conducted by Heber et al. was separated into 2 trials; The study conducted by Fuster-Munoz et al. was also separated into 2 trials 


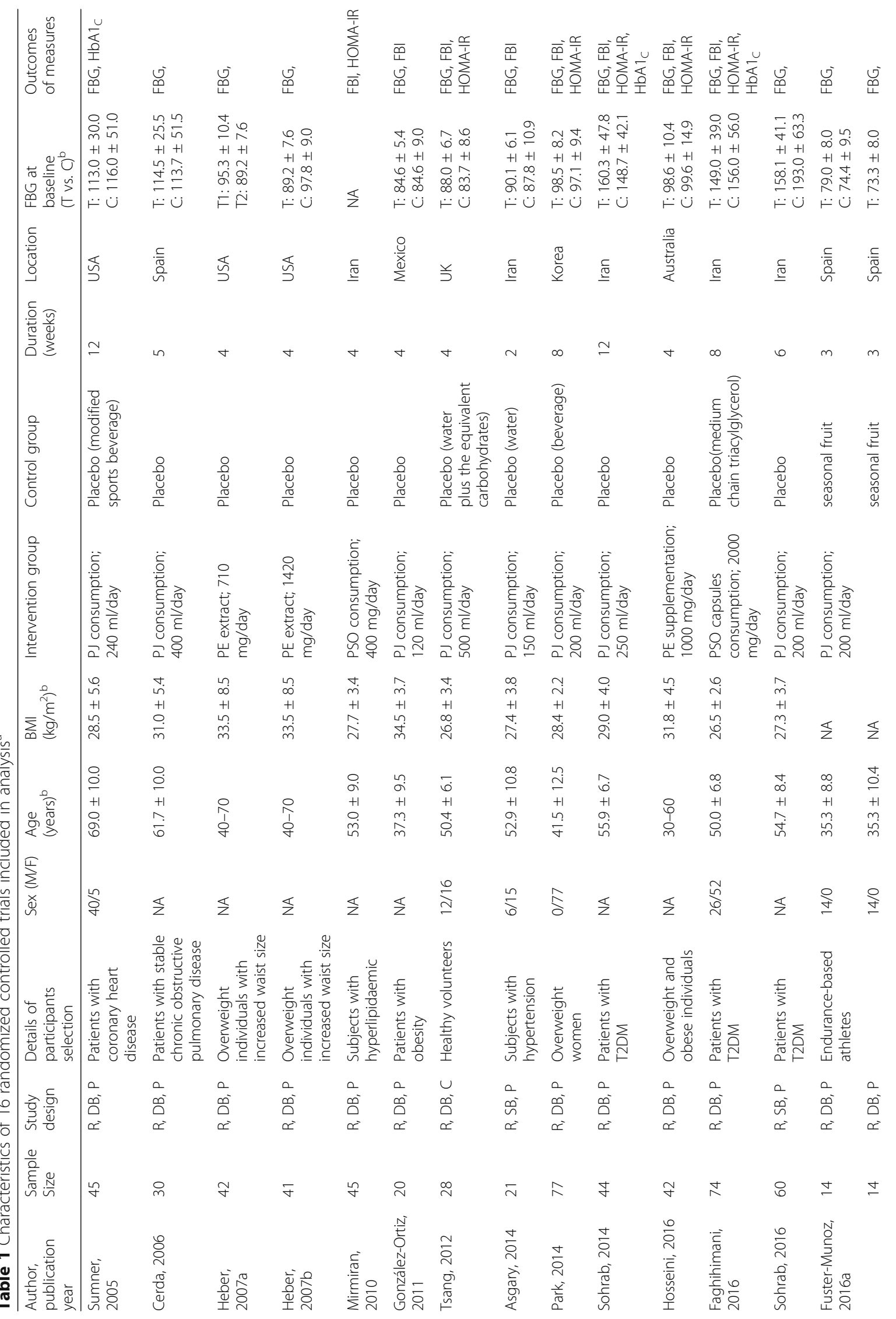




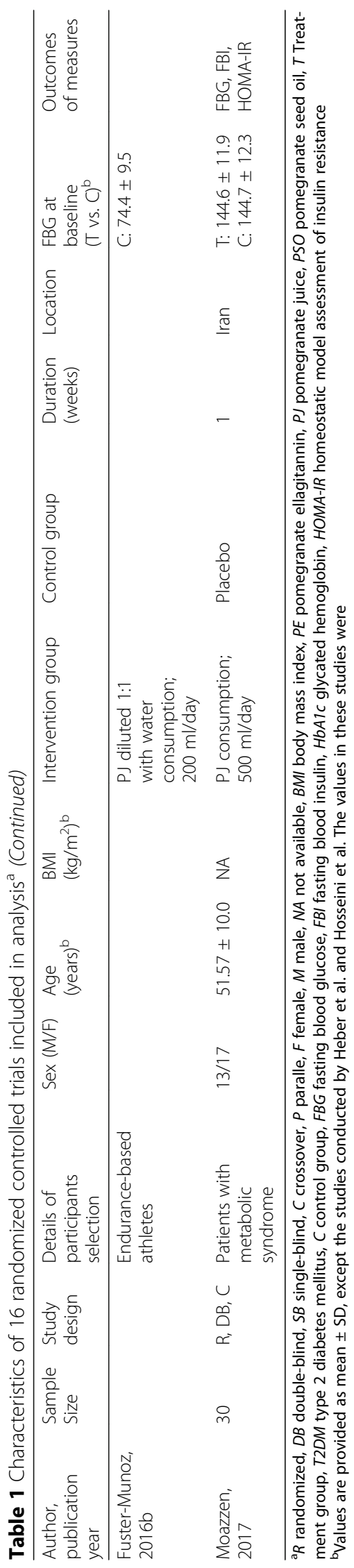


studies utilized pomegranate extract as the intervention (the dosage ranged from 710 to $1420 \mathrm{mg} /$ day) [39, 42]. The duration of the pomegranate intervention varied from 1 to 12 week (median: 5.5 week). Twelve of the 16 studies included subjects with a risk of cardiovascular disease, such as subjects with T2DM, overweight or obesity, hypertension, hyperlipidaemia and metabolic syndrome. Of the remaining 4 trials, one was conducted in patients with stable chronic obstructive pulmonary disease, and 3 studies were performed in healthy subjects. Fourteen trials adopted parallel study designs, and the 2 remaining trials used crossover designs. Fourteen trials were double-blind, placebo-controlled clinical trials. Among the included studies, investigators attempted to maintain the usual lifestyles of the participants. Details regarding the risk of bias assessment for the individual validity components are presented in Fig. 2. Overall, 5 trials were categorized as at low risk of bias, 2 as a high risk of bias, and 9 as unclear.

\section{Overall effect of pomegranate on glucose control and insulin sensitivity}

A total of 15 trials reported data on FBG concentrations. Compared with the control group, pomegranate intake did not significantly affect the FBG concentrations, and the pooled estimated change in FBG was $-0.60 \mathrm{mg} / \mathrm{dL}$ (95\% CI: $-2.79,1.58 ; P=0.59$ ) by the random-effects model (Fig. 3a). Eight trials reported data on fasting insulin concentrations, and the pooled estimated effect was $0.29 \mu \mathrm{IU} / \mathrm{mL}(95 \% \mathrm{CI}:-1.16,1.75 ; P=0.70)$ by the random-effects model (Fig. 3b). Seven studies reported the results on HOMA-IR, and the pooled results from the random-effects model for estimated mean difference was -0.04 (95\% CI: $-0.53,0.46 ; P=0.88$ ) (Fig. 3c). Three studies provided data on $\mathrm{HbA} 1 \mathrm{c}$, and pooling the data of these studies showed that pomegranate supplementation failed to show a significant effect on HbA1c (random-effects model; WMD, $-0.11 ; 95 \% \mathrm{CI},-0.39,0.18 ; P=0.46$; Fig. 3d). Between-study heterogeneity was observed in the effects of pomegranate on fasting insulin concentrations $\left(\mathrm{I}^{2}=60.4 \%\right)$ and HOMA-IR $\left(\mathrm{I}^{2}=59.8 \%\right)$, but not in HbA1c $\left(\mathrm{I}^{2}=0 \%\right)$ or FBG $\left(\mathrm{I}^{2}=0 \%\right)$ levels.

\section{Subgroup analyses and sensitivity analysis}

Subgroup analyses were performed to explore the effects of health status (CVD risk vs. healthy), study design (parallel vs. crossover), types of intervention (PJ supplementation vs. PE/POS supplementation), intervention duration ( $<5$ week. vs. $\geq 5$ week), baseline FBG levels $(<100 \mathrm{mg} / \mathrm{dL}$ vs. $100-126 \mathrm{mg} / \mathrm{dL}$ vs. $\geq 126 \mathrm{mg} / \mathrm{dL})$ and

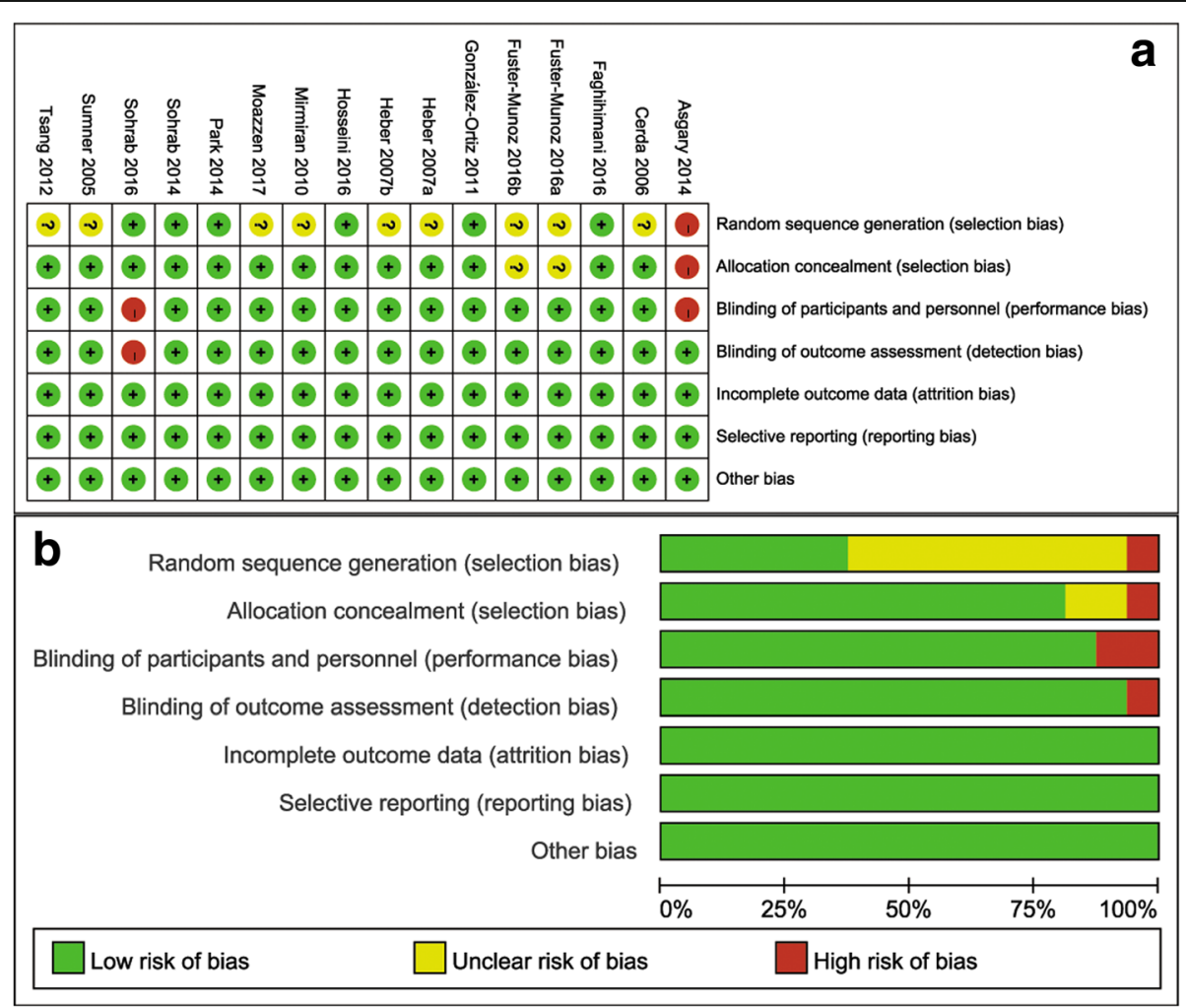

Fig. 2 Results of risk of bias assessment. a Risk of bias graph: review authors' judgments about each risk of bias item presented as percentages across all included studies. $\mathbf{b}$ Risk of bias summary: review authors' judgments about each risk of bias item for each included study 


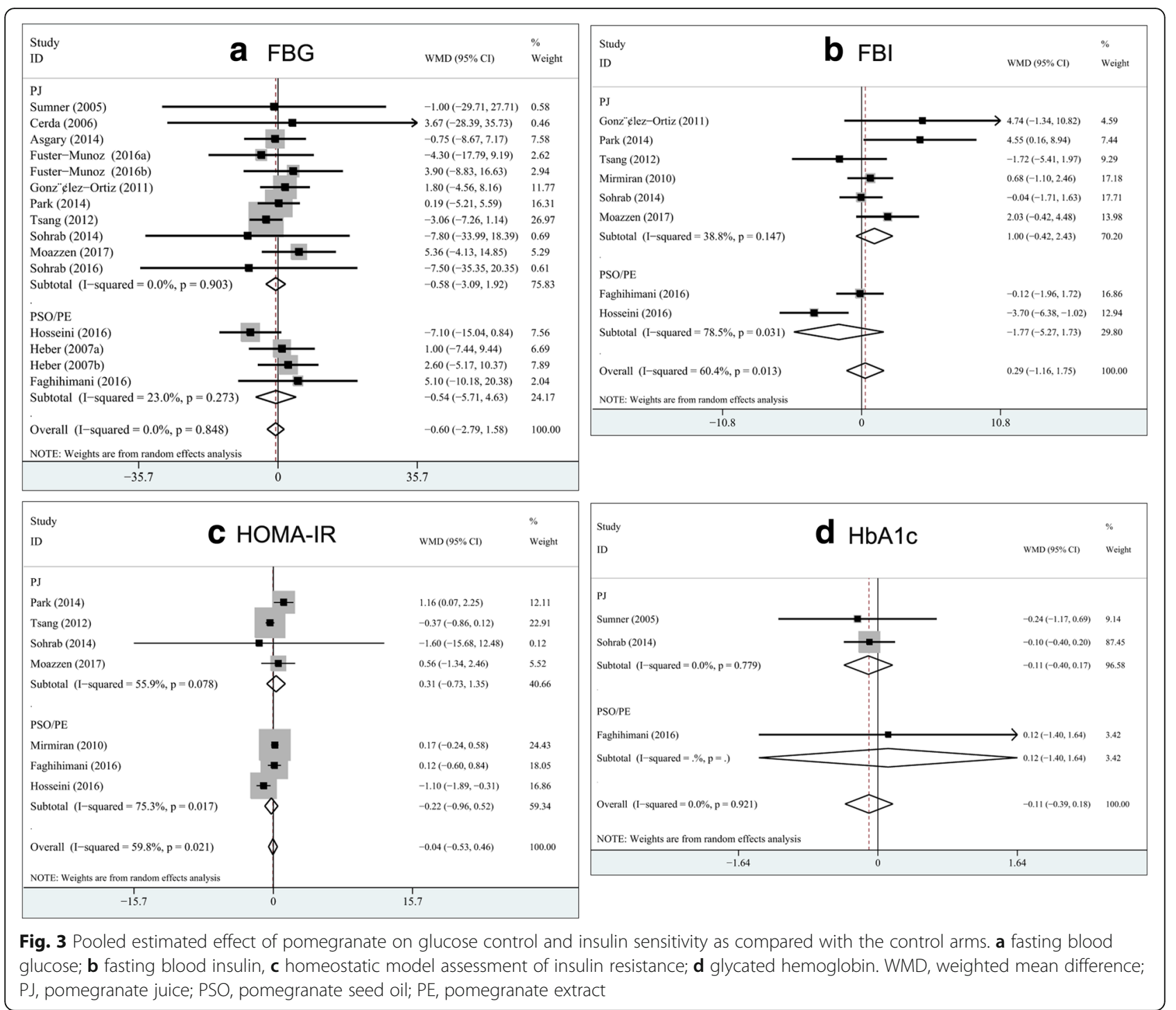

baseline BMI $\left(<30 \mathrm{~kg} / \mathrm{m}^{2}\right.$ vs. $\left.\geq 30 \mathrm{~kg} / \mathrm{m}^{2}\right)$ on the overall effects of pomegranate on fasting glucose and insulin concentrations, respectively.

The subgroup analysis results are summarized in Table 2. There were no statistically significant differences in the pooled effects of pomegranate on FBG in the subgroups stratified by study designs, intervention durations, types of intervention, baseline BMI, and baseline FBG levels. We also stratified the studies according to the type of patient, and no significant difference in the FBG-lowering effect was found between trials that were conducted in subjects with CVD risk (WMD, $0.30 \mathrm{mg} / \mathrm{dL}, 95 \% \mathrm{CI},-2.36$ to $2.97 ; P=0.82$ ) and those that were conducted in healthy individuals (WMD, $-2.53 \mathrm{mg} / \mathrm{dL}, 95 \% \mathrm{CI},-6.36$ to $1.30 ; P=0.19)$. Similarly, the subgroup analyses indicated that differences in study design, type of intervention, baseline BMI, and health status of the participants did not appear to significantly influence pooled mean differences in FBI concentrations.

To further confirm the robustness of the results, we conducted a sensitivity analysis. Exclusion of any individual study from the overall analysis did not meaningfully change the magnitude or direction of the summary effect of pomegranate on fasting glucose and insulin concentrations. Sensitivity analyses also showed that the aggregated results in FBG was not altered after removal of the 2 trials with a high risk of bias, and 1 trial did not use a placebo as a control (Table 2).

\section{Meta-regression analysis of the dose-response effect}

The meta-regression analyses were performed only for FBG because of the small numbers of studies including the other outcomes. Here, random-effects metaregression analysis suggested that no significant 
Table 2 Subgroup analyses of fasting glucose and insulin concentrations stratified by previously defined study characteristics

\begin{tabular}{|c|c|c|c|c|c|c|c|c|}
\hline \multirow[t]{2}{*}{ Variables } & \multicolumn{4}{|c|}{ Fasting glucose } & \multicolumn{4}{|c|}{ Fasting insulin } \\
\hline & No. of trials & Net change $(95 \% \mathrm{Cl})^{\mathrm{a}}$ & $P^{*}$ & $\begin{array}{l}P^{2} \\
(\%)^{b}\end{array}$ & No. of trials & Net change $(95 \% \mathrm{Cl})^{\mathrm{a}}$ & $P^{*}$ & $\begin{array}{l}P^{2} \\
(\%)^{b}\end{array}$ \\
\hline \multicolumn{9}{|l|}{ Sensitivity analyses } \\
\hline Exclude high-risk research & 13 & $-0.55(-2.82$ to 1.73$)$ & 0.64 & 0 & 8 & $0.29(-1.16$ to 1.75$)$ & 0.70 & 60 \\
\hline $\begin{array}{l}\text { Removing study did not use } \\
\text { placebo as control }\end{array}$ & 13 & $-0.64(-2.89$ to 1.60$)$ & 0.58 & 0 & 8 & $0.29(-1.16$ to 1.75$)$ & 0.70 & 60 \\
\hline \multicolumn{9}{|l|}{ Subgroup analyses } \\
\hline \multicolumn{9}{|l|}{ Study design } \\
\hline Parallel & 13 & $-0.09(-2.74$ to 2.56$)$ & 0.95 & 0 & 6 & $0.24(-1.49$ to 1.97$)$ & 0.78 & 65 \\
\hline Crossover & 2 & $0.03(-7.92$ to 7.98$)$ & 0.99 & 60 & 2 & $0.42(-3.22$ to 4.06$)$ & 0.82 & 64 \\
\hline \multicolumn{9}{|l|}{ Duration } \\
\hline$<5$ week & 9 & $-0.82(-3.27$ to 1.63$)$ & 0.51 & 0 & 5 & $0.01(-2.40$ to 2.42$)$ & 0.99 & 71 \\
\hline$\geq 5$ week & 6 & $0.22(-4.57$ to 5.02$)$ & 0.93 & 0 & 3 & $0.58(-1.28$ to 2.43$)$ & 0.54 & 49 \\
\hline \multicolumn{9}{|l|}{ Type of intervention } \\
\hline PJ consumption & 11 & $-0.58(-3.09$ to 1.92$)$ & 0.65 & 0 & 5 & $1.28(-0.74$ to 3.30$)$ & 0.22 & 51 \\
\hline$<250 \mathrm{ml} / \mathrm{d}$ & 7 & $0.32(-3.03$ to 3.67$)$ & 0.85 & 0 & 3 & 2.86 (0.00 to 4.88$)$ & 0.05 & 0 \\
\hline$\geq 250 \mathrm{ml} / \mathrm{d}$ & 4 & $-1.73(-5.51$ to 2.04$)$ & 0.37 & 0 & 2 & $-0.33(-1.85$ to 1.20$)$ & 0.67 & 0 \\
\hline PE/PSO consumption & 4 & $-0.67(-5.10$ to 3.77$)$ & 0.77 & 23 & 3 & $-0.84(-3.12$ to 1.44$)$ & 0.47 & 73 \\
\hline$\leq 1000 \mathrm{mg} / \mathrm{d}$ & 2 & -3.30 (-9.08 to 2.48$)$ & 0.26 & 47 & 2 & $-1.39(-5.68$ to 2.90$)$ & 0.52 & 86 \\
\hline$>1000 \mathrm{mg} / \mathrm{d}$ & 2 & 3.31 (-3.81 to 10.04$)$ & 0.38 & 0 & 1 & $-0.12(-1.96$ to 1.72$)$ & 0.9 & NA \\
\hline \multicolumn{9}{|l|}{ Healthy status } \\
\hline CVD risk & 11 & $0.30(-2.36$ to 2.97$)$ & 0.82 & 0 & 7 & $0.51(-1.05$ to 2.07$)$ & 0.52 & 64 \\
\hline Healthy & 3 & $-2.53(-6.36$ to 1.30$)$ & 0.19 & 0 & 1 & $-1.72(-5.41$ to 1.97$)$ & 0.36 & NA \\
\hline \multicolumn{9}{|l|}{ BMI } \\
\hline$<30 \mathrm{~kg} / \mathrm{m}^{2}$ & 7 & $-1.56(-4.51$ to 1.39$)$ & 0.30 & 0 & 5 & $0.29(-0.87$ to 1.44$)$ & 0.63 & 24 \\
\hline$\geq 30 \mathrm{~kg} / \mathrm{m}^{2}$ & 5 & $-0.10(-3.83$ to 3.62$)$ & 0.96 & 0 & 2 & $0.06(-8.16$ to 8.28$)$ & 0.99 & 84 \\
\hline \multicolumn{9}{|l|}{ Baseline FBG levels } \\
\hline$<100 \mathrm{mg} / \mathrm{dL}$ & 9 & $-1.00(-3.29$ to 1.30$)$ & 0.39 & 0 & 4 & $0.51(-3.74$ to 4.76$)$ & 0.81 & 78 \\
\hline $100-126 \mathrm{mg} / \mathrm{dL}$ & 2 & $1.08(-20.31$ to 22.47$)$ & 0.92 & 0 & 0 & - & - & - \\
\hline$\geq 126 \mathrm{mg} / \mathrm{dL}$ & 4 & $3.33(-4.1$ to 10.75$)$ & 0.38 & 0 & 3 & $0.38(-0.81$ to 1.56$)$ & 0.53 & 12 \\
\hline
\end{tabular}

${ }^{a}$ Net change was expressed as weighted mean difference

${ }^{b}$ The $I^{2}$ statistic was calculated by using Cochran's test, and $I^{2}>50 \%$ was considered to indicate significant heterogeneity across studies

${ }^{*} P$ for meta-analysis: $P<0.05$ was considered to indicate a significant effect of pomegranate on fasting glucose and insulin concentrations by using a

random-effects model

association was observed between the duration of pomegranate supplementation and mean differences in glucose levels (coefficient, $-0.119 ; 95 \% \mathrm{CI},-1.29$ to $1.06 ; P=0.83$ ). Moreover, our meta-regression analysis showed that the factor (dose of pomegranate juice supplementation) was not associated with the treatment effects on FBG level (coefficient, $-0.006 ; 95 \% \mathrm{CI},-0.023$ to $0.011, P=0.46$ ). The results of the meta-regression analyses are shown in Fig. 4.

\section{Publication bias and GRADE profile evidence}

No evidence of publication bias was found in the present meta-analysis (for both primary and secondary outcomes). The funnel plots were symmetrical (Fig. 5), and
Egger's regression test suggested no significant asymmetry for the overall effect estimation on the study endpoints (for FBG, $P=0.96$; for FBI, $P=0.62$; for HOMA-IR, $P=0.88$; for HbA1c, $P=0.60$ ). The GRADE evidence profiles for the primary and secondary outcomes are presented as Additional file 1. The GRADE Working Group grades of the level of evidence are high for FBG and HbA1c and moderate for FBI and HOMA-IR.

\section{Discussion}

The findings from the current study demonstrate that pomegranate intake did not lead to any significant changes in circulating glucose levels, insulin concentrations, 

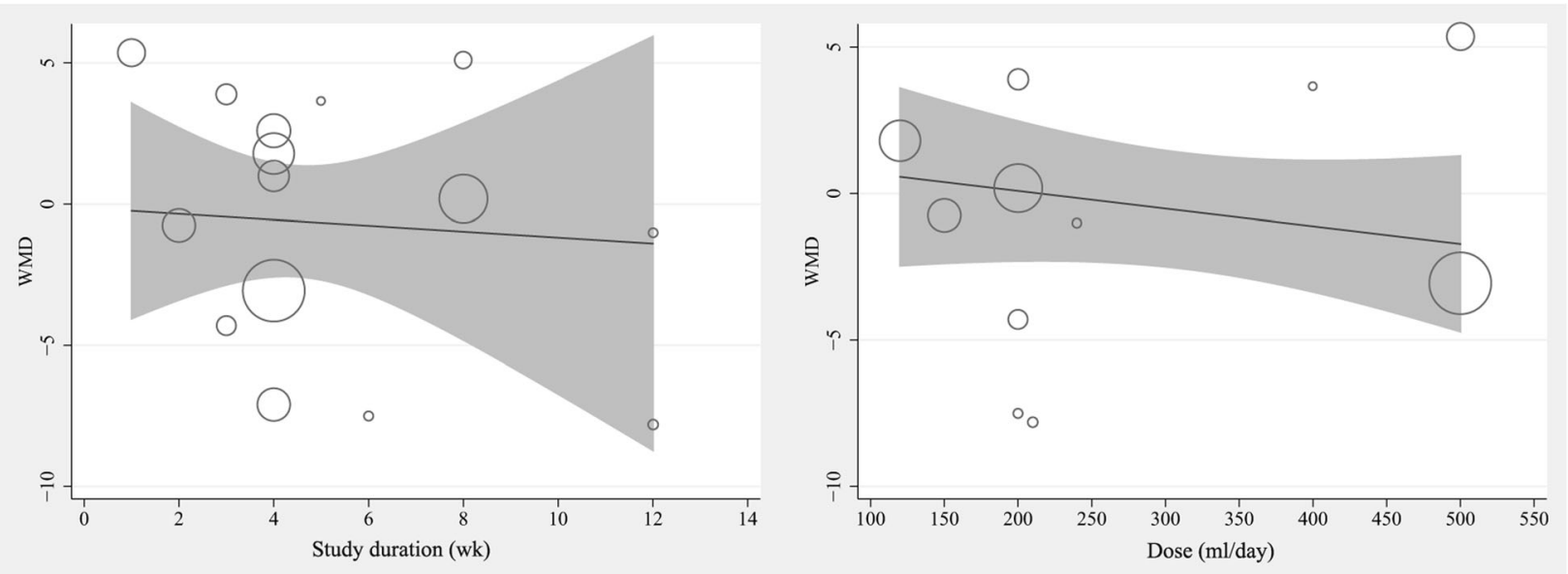

Fig. 4 Meta-regression result of the association between mean changes in fasting glucose concentrations with dose and duration of supplementation with pomegranate juice
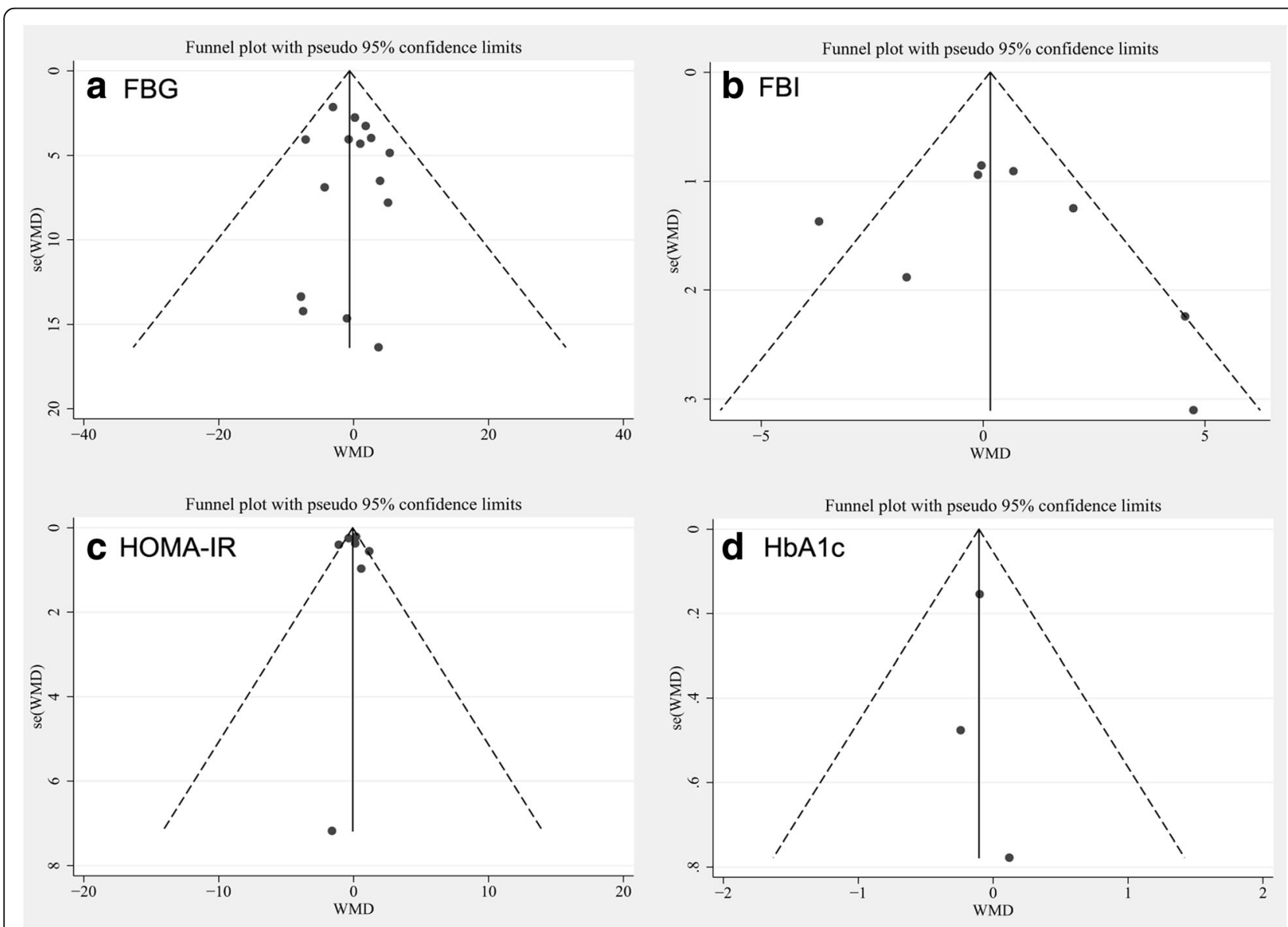

Fig. 5 Tests for publication bias of impact of pomegranate consumption on glucose control and insulin sensitivity. a FBG, fasting blood glucose; b FBl, fasting blood insulin; c HOMA-IR, homeostatic model assessment of insulin resistance; $\mathbf{d} \mathrm{HbA1C}$, glycated hemoglobin 
HOMA-IR or HbA1c. The target population for T2DM prevention has been a topic of debate since the completion of major diabetes prevention trials [47]. The difficulty stems from observations that diabetes prevalence has increased across all segments of society [48]. However, the evidence for preventive interventions is mainly limited to persons with impaired glucose tolerance (IGT) [49]. Assessment of glycemic control is a key element of diabetes care. Reliable information about glycemic variations allows physicians and patients to evaluate the effect of treatment on restoration and maintenance of blood glucose to within the physiologic range.

Dietary micronutrients or herbal medication interventions have generally been used to improve glycemic control and other CVD risk factors among individuals with T2DM, and the public has embraced their efficacy and safety $[11,50,51]$. A recent meta-analysis reported that consumption of cinnamon is associated with a statistically significant decrease in the levels of FBG, total cholesterol, LDL-C, and triglyceride levels and an increase in HDL-C levels [11]. Potential side effects reported to be associated with cinnamon were hepatotoxicity, decreased platelet counts, increases in the risk of bleeding, and markedly increased allergy/hypersensitivity. However, human studies suggested that no significant side effects were seen with cinnamon use. Hausenblas et al. [50] found that resveratrol supplementation was more effective than placebo in controlling systolic blood pressure, $\mathrm{HbA1c}$, and creatinine. The incidence of side effects was very small and not different from placebo, and no major adverse events were reported.

Over the last decade, various studies have linked pomegranate and its active compounds with diabetes prevention and treatment. The study conducted by Huang et al. demonstrated that treatment with Punica granatum flower extract could enhanced the mRNA expression of cardiac PPAR- $\gamma$ and restore the mRNA expression of the cardiac glucose transporter 4 (GLUT4) [52]. Later reports have shown that PSO reduced the risk for T2DM in wildtype CD-1 mice by improving insulin sensitivity [53]. Pomegranate flowers also ameliorate T2DM in Zucker fatty diabetic rats by enhancing the expression of hepatic genes involved in fatty acid oxidation (e.g., acyl-CoA oxidase and carnitine palmitoyl-transferase-1) [54]. Furthermore, pomegranate extract is beneficial in controlling glucose homeostasis in humans by suppressing the activation of NF- $\mathrm{kB}$, neutralizing the generated reactive oxygen species and the expression of tumour necrosis factor- $\alpha$, which finally delays the development of T2DM $[55,56]$.

However, data from human clinical trials that have evaluated the possible beneficial effects of pomegranate products and extracts on glycemic control and insulin sensitivity have generated mixed findings [27-32]. In the present study, we aim to provide a focused and comprehensive evaluation of the precise effects of pomegranate supplementation on measures of glycaemic control and insulin sensitivity in humans. A total of 16 RCTs were included in the analysis, and methodological quality assessment suggested that the overall data quality was fair. The Egger's regression test's symmetry testing of the funnel plot did not indicate a notable publication bias for the overall effect estimation of WMDs in the primary outcomes. The results remain robust and consistent when pre-specified defined subgroup analyses were conducted. Meta-regression analysis also revealed the absence of any dose-response relation between the pomegranate juice intake and any effect on FBG concentration. Moreover, exclusion of any single study and sensitivity analyses based on various exclusion criteria (i.e., removal of the trials with a high risk of bias and the trials that did not use a placebo as a control) did not materially alter the pooled results, which adds robustness to our main results. The magnitude of WMDs reported in highquality studies was stronger than the magnitude reported in the overall analysis $(0.55 \mathrm{mg} / \mathrm{dL}$ compared with $0.06 \mathrm{mg} / \mathrm{dL}$ ), which indicated that the real effects may be influenced by poor study methodologies.

Several methods, each with differing utility and limitations, exist for monitoring glycemic control [57]. Fasting plasma glucose levels are considered a key variable in the diagnosis of diabetes and are also adopted by the FDA to evaluate the efficacy of dietary supplements and drugs. Among the included studies, most studies reported only fasting glucose (93.7\%) and fasting insulin (50\%) concentrations, and there was a lack of other important variables for glycemic control and insulin sensitivity. Previous data indicated that glucose and insulin concentrations fluctuate with changes after diet, exercise, and use of some medications. Glycated protein, such as $\mathrm{HbA1c}$, is considered the standard measure of long-term glycemic control, and the measurement of $\mathrm{HbA}_{1 \mathrm{c}}$ levels is strongly associated with complications of diabetes. In the present study, however, we failed to find any statistically significant differences between the relation between dietary pomegranate supplementation and reductions in HbA1c (WMD, $-0.11 \%, 95 \% \mathrm{CI},-0.39$ to $0.18, P=0.46$ ). Most notably, these results are inconclusive because of the limited eligible RCTs included in these outcomes (only 3 studies reported HbAlc concentrations); further large-scale and well-performed studies that report a more comprehensive set of indicators for glycemic control and insulin sensitivity are needed.

Our meta-analysis has several strengths. The present analysis was restricted to RCTs that met predetermined methodological criteria to minimize the potential for bias. The relatively large number of pooled participants allowed us a greater statistical power to detect a small treatment effect. To increase the robustness of our 
study, we performed several subgroup analyses and sensitivity analysis. Although we had limited this study to well-designed RCTs and performed quality assessment to reduce the possible selective bias, the present meta-analysis still had several caveats that could affect the interpretation of the results. First, although extensive searches with clear inclusion criteria were performed, it cannot be entirely guaranteed that all relevant articles were included, since the measures of blood glucose or insulin were not primary outcomes in the trials selected for this meta-analysis, and the null findings of secondary outcomes may not always have been published. Second, there was unavoidable heterogeneity across the studies for FBI and HOMA-IR outcomes $\left(\mathrm{I}^{2}=60.4 \%\right.$ and $59.8 \%$, respectively). This heterogeneity may have been due to study differences in design, study population and characteristics, and duration of treatment. Regardless of the cause, the pooled multivariate estimates were managed using a random-effects model, which could reduce the bias to some extent. Third, our pooled results were based on unadjusted estimates; the precise effect of pomegranate on diabetes biomarkers could be impacted by various confounders (i.e., other lifestyle interventions or smoking habits). The synergistic effects of other coexisting substances on the clinical outcomes need to be excluded during the study period. Fourth, due to the limited number of DM patients included, the overall effects of pomegranate on parameters of glucose metabolism are inconclusive; additional adequately powered studies investigating the effects of pomegranate on the biomarkers of glucose metabolism in adults with DM are needed. Finally, the intervention durations of the included studies were relatively short (ranging from 1 to 12 weeks with a median of 5.5 weeks), and the more long-term durability of the pomegranate treatment therefore is unknown. Finally, a standardized protocol of pomegranate consumption (such as consistency regarding dosage, route, timing, and duration of the intervention) are needed in the future.

\section{Conclusions}

In conclusion, pomegranate intake did not show notably favourable effects on improvements in glucose and insulin metabolism. The current evidence suggests that daily pomegranate supplementation is not recommended as a potential therapeutic strategy in glycemic and insulin management. Additional well-reported RCTs that are specifically designed to evaluate the effect of pomegranate or pomegranate extracts on a set of comprehensive clinical outcomes related to glycemic control are required.

\section{Additional file}

Additional file 1: GRADE Evidence Profile. (DOC $38 \mathrm{~kb}$ )

\begin{abstract}
Abbreviations
Cl: Confidence interval; CVD: Cardiovascular disease; FBG: Fasting blood glucose; FBI: Fasting blood insulin; HbA1c: Glycated hemoglobin; HOMAIR: Homeostatic model assessment of insulin resistance; IFG: Impaired fasting glucose; IGT: Impaired glucose tolerance; MeSH: Medical Subject Headings; PE: Pomegranate extract; PJ: Pomegranate juice; PRISMA: Preferred Reporting Items for Systematic Reviews and Meta-Analyses; PSO: Pomegranate seed oil; RCTs: Randomized controlled trials; SEs: Standard errors; T2DM: Type 2 diabetes mellitus; WMD: Weighted mean difference
\end{abstract}

\section{Acknowledgements}

Not applicable.

Funding

Not applicable.

Availability of data and materials

Please contact author for data requests.

\section{Declarations}

Not applicable.

\section{Authors' contributions}

$\mathrm{HH}$ : Conception and design of the study, acquisition of data, analysis and interpretation of data, discussed the idea of the meta-analysis, drafted the manuscript, submitted the paper. DL and GC: Completed the database searches and selected, screened, and reviewed the articles. HC and YZ: reviewed and extracted the data, and performed the data analyses. All authors read and approved the final manuscript. HH had primary

responsibility for final content.

Ethics approval and consent to participate

Not applicable.

Consent for publication

Not applicable.

Competing interests

The authors declare that they have no competing interests.

\section{Publisher's Note}

Springer Nature remains neutral with regard to jurisdictional claims in published maps and institutional affiliations.

\section{Author details}

1Department of Clinical Pharmacy, Dongguan Third People's Hospital, Affiliated Dongguan Shilong People's Hospital of Southern Medical University, Dongguan, Guangdong, China. ²Department of Gynaecology and Obstetric, Dongguan Third People's Hospital, Affiliated Dongguan Shilong People's Hospital of Southern Medical University, Dongguan, Guangdong, China. ${ }^{3}$ Department of Pharmacy, Guangdong Province Agricultural Reclamation Central Hospital, Zhanjiang, Guangdong, China. ${ }^{4}$ School of Pharmacy, Guangdong Medical University, Dongguan, Guangdong, China.

Received: 12 June 2017 Accepted: 26 September 2017

Published online: 06 October 2017

\section{References}

1. Whiting DR, Guariguata L, Weil C, Shaw J. IDF diabetes atlas: global estimates of the prevalence of diabetes for 2011 and 2030. Diabetes Res Clin Pract. 2011;94:311-21.

2. Woodward M, Zhang X, Barzi F, Pan W, Ueshima H, Rodgers A, MacMahon $S$. The effects of diabetes on the risks of major cardiovascular diseases and death in the Asia-Pacific region. Diabetes Care. 2003;26:360-6. 
3. Ciulla TA, Amador AG, Zinman B. Diabetic retinopathy and diabetic macular edema: pathophysiology, screening, and novel therapies. Diabetes Care. 2003;26:2653-64

4. Jha V, Garcia-Garcia G, Iseki K, Li Z, Naicker S, Plattner B, Saran R, Wang AY, Yang CW. Chronic kidney disease: global dimension and perspectives. Lancet. 2013;382:260-72.

5. Gerstein HC, Santaguida P, Raina P, Morrison KM, Balion C, Hunt D, Yazdi H, Booker L. Annual incidence and relative risk of diabetes in people with various categories of dysglycemia: a systematic overview and meta-analysis of prospective studies. Diabetes Res Clin Pract. 2007;78:305-12.

6. Hanley AJ, Wagenknecht LE, Norris JM, Bryer-Ash M, Chen YI, Anderson AM, Bergman R, Haffner SM. Insulin resistance, beta cell dysfunction and visceral adiposity as predictors of incident diabetes: the Insulin Resistance Atherosclerosis Study (IRAS) Family study. Diabetologia. 2009;52:2079-86.

7. Shimodaira M, Okaniwa S, Nakayama T. Investigation of the relationship between hemoglobin and serum iron levels and early-phase insulin secretion in non-diabetic subjects. Acta Diabetol. 2016;53:783-9.

8. Krisai P, Leib S, Aeschbacher S, Kofler T, Assadian M, Maseli A, Todd J, Estis J, Risch M, Risch L, Conen D. Relationships of iron metabolism with insulin resistance and glucose levels in young and healthy adults. Eur J Intern Med. 2016;32:31-7.

9. Liu Y, Li J, Wang T, Wang Y, Zhao L, Fang Y. The effect of genistein on glucose control and insulin sensitivity in postmenopausal women: A meta-analysis. Maturitas. 2017;97:44-52.

10. Liu K, Zhou R, Wang B, Chen K, Shi LY, Zhu JD, Mi MT. Effect of green tea on glucose control and insulin sensitivity: a meta-analysis of 17 randomized controlled trials. Am J Clin Nutr. 2013;98:340-8.

11. Allen RW, Schwartzman E, Baker WL, Coleman Cl, Phung OJ. Cinnamon use in type 2 diabetes: an updated systematic review and meta-analysis. Ann Fam Med. 2013;11:452-9.

12. Medjakovic $S$, Jungbauer A. Pomegranate: a fruit that ameliorates metabolic syndrome. Food Funct. 2013:4:19-39.

13. Ismail T, Sestili P, Akhtar S. Pomegranate peel and fruit extracts: a review of potential anti-inflammatory and anti-infective effects. J Ethnopharmacol. 2012;143:397-405.

14. Krueger DA. Composition of pomegranate juice. J AOAC Int. 2012;95:163-8.

15. Basu A, Penugonda K. Pomegranate juice: a heart-healthy fruit juice. Nutr Rev. 2009:67:49-56.

16. Arao K, Wang YM, Inoue N, Hirata J, Cha JY, Nagao K, Yanagita T. Dietary effect of pomegranate seed oil rich in 9cis, 11trans, 13cis conjugated linolenic acid on lipid metabolism in obese, hyperlipidemic OLETF rats. Lipids Health Dis. 2004;3:24

17. Li Y, Guo C, Yang J, Wei J, Xu J, Cheng S. Evaluation of antioxidant properties of pomegranate peel extract in comparison with pomegranate pulp extract. Food Chem. 2006;96:254-60

18. Das AK, Mandal SC, Banerjee SK, Sinha S, Saha BP, Pal M. Studies on the hypoglycaemic activity of Punica granatum seed in streptozotocin induced diabetic rats. Phytother Res. 2001;15:628-9.

19. Jurenka JS. Therapeutic applications of pomegranate (Punica granatum L.): a review. Altern Med Rev. 2008;13:128-44.

20. Sahebkar A, Ferri C, Giorgini P, Bo S, Nachtigal P, Grassi D. Effects of pomegranate juice on blood pressure: A systematic review and metaanalysis of randomized controlled trials. Pharmacol Res. 2017;115:149-61.

21. Parmar HS, Kar A. Antidiabetic potential of Citrus sinensis and Punica granatum peel extracts in alloxan treated male mice. Biofactors. 2007;31:17-24

22. Bagri P, Ali M, Aeri V, Bhowmik M, Sultana S. Antidiabetic effect of Punica granatum flowers: effect on hyperlipidemia, pancreatic cells lipid peroxidation and antioxidant enzymes in experimental diabetes. Food Chem Toxicol. 2009;47:50-4.

23. Vroegrijk IO, van Diepen JA, van den Berg S, Westbroek I, Keizer H, Gambelli L, Hontecillas R, Bassaganya-Riera J, Zondag GC, Romijn JA, et al. Pomegranate seed oil, a rich source of punicic acid, prevents diet-induced obesity and insulin resistance in mice. Food Chem Toxicol. 2011;49:1426-30.

24. Rock W, Rosenblat M, Miller-Lotan R, Levy AP, Elias M, Aviram M. Consumption of wonderful variety pomegranate juice and extract by diabetic patients increases paraoxonase 1 association with high-density lipoprotein and stimulates its catalytic activities. J Agric Food Chem. 2008;56:8704-13.

25. Katz SR, Newman RA, Lansky EP. Punica granatum: heuristic treatment for diabetes mellitus. J Med Food. 2007;10:213-7.

26. Fenercioglu AK, Saler T, Genc E, Sabuncu H, Altuntas $Y$. The effects of polyphenol-containing antioxidants on oxidative stress and lipid peroxidation in Type 2 diabetes mellitus without complications. Endocrinol Investig. 2010;33:118-24.

27. Sumner MD, Elliott-Eller M, Weidner G, Daubenmier JJ, Chew MH, Marlin R, Raisin CJ, Ornish D. Effects of pomegranate juice consumption on myocardial perfusion in patients with coronary heart disease. Am J Cardiol. 2005:96:810-4.

28. Cerda B, Soto C, Albaladejo MD, Martinez P, Sanchez-Gascon F, TomasBarberan F, Espin JC. Pomegranate juice supplementation in chronic obstructive pulmonary disease: a 5-week randomized, double-blind, placebo-controlled trial. Eur J Clin Nutr. 2006;60:245-53.

29. Mirmiran P, Fazeli MR, Asghari G, Shafiee A, Azizi F. Effect of pomegranate seed oil on hyperlipidaemic subjects: a double-blind placebo-controlled clinical trial. Br J Nutr. 2010;104:402-6.

30. Gonzalez-Ortiz M, Martinez-Abundis E, Espinel-Bermudez MC, Perez-Rubio KG. Effect of pomegranate juice on insulin secretion and sensitivity in patients with obesity. Ann Nutr Metab. 2011;58:220-3.

31. Tsang C, Smail NF, Almoosawi S, Davidson I, Al-Dujaili EA. Intake of polyphenol-rich pomegranate pure juice influences urinary glucocorticoids, blood pressure and homeostasis model assessment of insulin resistance in human volunteers. J Nutr Sci. 2012;1:e9.

32. Asgary S, Sahebkar A, Afshani MR, Keshvari M, Haghjooyjavanmard S, RafieianKopaei M. Clinical evaluation of blood pressure lowering, endothelial function improving, hypolipidemic and anti-inflammatory effects of pomegranate juice in hypertensive subjects. Phytother Res. 2014;28:193-9.

33. Liberati A, Altman DG, Tetzlaff J, Mulrow C, Gotzsche PC, loannidis JP, Clarke M, Devereaux PJ, Kleijnen J, Moher D. The PRISMA statement for reporting systematic reviews and meta-analyses of studies that evaluate health care interventions: explanation and elaboration. PLoS Med. 2009:6:e1000100.

34. Higgins JP, Green S: Cochrane Handbook for System-atic Reviews of Interventions Version 5.1.0. (updated March 2011). Available at: http:// handbook.cochrane.org/.

35. Higgins JP, Thompson SG, Deeks JJ, Altman DG. Measuring inconsistency in meta-analyses. BMJ. 2003;327:557-60

36. Nikolakopoulou A, Mavridis D, Salanti G. Demystifying fixed and random effects meta-analysis. Evid Based Ment Health. 2014;17:53-7.

37. Egger M, Davey Smith G, Schneider M, Minder C. Bias in meta-analysis detected by a simple, graphical test. BMJ. 1997;315:629-34.

38. Morton SC, Adams JL, Suttorp MJ, Shekelle PG: Meta-regression Approaches: What, Why, When, and How? Rockville MD2004.

39. Heber D, Seeram NP, Wyatt $H$, Henning SM, Zhang Y, Ogden LG, Dreher M, Hill JO. Safety and antioxidant activity of a pomegranate ellagitanninenriched polyphenol dietary supplement in overweight individuals with increased waist size. J Agric Food Chem. 2007;55:10050-4.

40. Fuster-Munoz E, Roche E, Funes L, Martinez-Peinado P, Sempere JM, Vicente-Salar N. Effects of pomegranate juice in circulating parameters, cytokines, and oxidative stress markers in endurance-based athletes: A randomized controlled trial. Nutrition. 2016:32:539-45.

41. Sohrab G, Nasrollahzadeh J, Zand H, Amiri Z, Tohidi M, Kimiagar M. Effects of pomegranate juice consumption on inflammatory markers in patients with type 2 diabetes: A randomized, placebo-controlled trial. J Res Med Sci. 2014;19:215-20.

42. Hosseini B, Saedisomeolia A, Wood LG, Yaseri M, Tavasoli S. Effects of pomegranate extract supplementation on inflammation in overweight and obese individuals: A randomized controlled clinical trial. Complement Ther Clin Pract. 2016;22:44-50.

43. Faghihimani Z, Mirmiran P, Sohrab G, Iraj B, Faghihimani E. Effects of Pomegranate Seed Oil on Metabolic State of Patients with Type 2 Diabetes Mellitus. Int J Prev Med. 2016;7:124.

44. Sohrab G, Ebrahimof S, Sotoudeh G, Neyestani TR, Angoorani P, Hedayati M, Siasi F. Effects of pomegranate juice consumption on oxidative stress in patients with type 2 diabetes: a single-blind, randomized clinical trial. Int J Food Sci Nutr. 2017:68:249-55.

45. Moazzen H, Alizadeh M. Effects of Pomegranate Juice on Cardiovascular Risk Factors in Patients with Metabolic Syndrome: a Double-Blinded, Randomized Crossover Controlled Trial. Plant Foods Hum Nutr. 2017;72:126-33.

46. Park J, Kim J, Kim J, Kim Y, Kin M, Kwon, Kwon O. Pomegranate vinegar beverage reduces visceral fat accumulation in association with AMPK activation in overweight women: A double-blind, randomized, and placebocontrolled trial. J Funct Foods. 2014;8:274-81.

47. Yudkin JS, Montori VM. The epidemic of pre-diabetes: the medicine and the politics. BMJ. 2014;349:94485. 
48. Geiss LS, Wang J, Cheng YJ, Thompson TJ, Barker L, Li Y, Albright AL, Gregg EW. Prevalence and incidence trends for diagnosed diabetes among adults aged 20 to 79 years, United States, 1980-2012. JAMA. 2014;312:1218-26.

49. Sakane N, Sato J, Tsushita K, Tsujii S, Kotani K, Tsuzaki K, Tominaga M, Kawazu S, Sato Y, Usui T, et al. Prevention of type 2 diabetes in a primary healthcare setting: three-year results of lifestyle intervention in Japanese subjects with impaired glucose tolerance. BMC Public Health. 2011;11:40.

50. Hausenblas HA, Schoulda JA, Smoliga JM. Resveratrol treatment as an adjunct to pharmacological management in type 2 diabetes mellitussystematic review and meta-analysis. Mol Nutr Food Res. 2015;59:147-59.

51. Sood N, Baker WL, Coleman Cl. Effect of glucomannan on plasma lipid and glucose concentrations, body weight, and blood pressure: systematic review and meta-analysis. Am J Clin Nutr. 2008:88:1167-75.

52. Huang TH, Peng G, Kota BP, Li GQ, Yamahara J, Roufogalis BD, Li Y. Antidiabetic action of Punica granatum flower extract: activation of PPARgamma and identification of an active component. Toxicol Appl Pharmacol. 2005:207:160-9.

53. McFarlin BK, Strohacker KA, Kueht ML. Pomegranate seed oil consumption during a period of high-fat feeding reduces weight gain and reduces type 2 diabetes risk in CD-1 mice. Br J Nutr. 2009;102:54-9.

54. KZ X, Zhu C, Kim MS, Yamahara J, Li Y. Pomegranate flower ameliorates fatty liver in an animal model of type 2 diabetes and obesity. J Ethnopharmacol. 2009;123:280-7.

55. Huang TH, Yang Q, Harada M, Li GQ, Yamahara J, Roufogalis BD, Li Y. Pomegranate flower extract diminishes cardiac fibrosis in Zucker diabetic fatty rats: modulation of cardiac endothelin-1 and nuclear factor-kappaB pathways. J Cardiovasc Pharmacol. 2005;46:856-62.

56. Fuhrman B, Volkova N, Aviram M. Pomegranate juice polyphenols increase recombinant paraoxonase-1 binding to high-density lipoprotein: studies in vitro and in diabetic patients. Nutrition. 2010;26:359-66.

57. Goldstein DE, Little RR, Lorenz RA, Malone Jl, Nathan DM, Peterson CM. Tests of glycemia in diabetes. Diabetes Care. 2004;27(Suppl 1):S91-3.

\section{Submit your next manuscript to BioMed Central and we will help you at every step:}

- We accept pre-submission inquiries

- Our selector tool helps you to find the most relevant journal

- We provide round the clock customer support

- Convenient online submission

- Thorough peer review

- Inclusion in PubMed and all major indexing services

- Maximum visibility for your research

Submit your manuscript at www.biomedcentral.com/submit 